\title{
Creep and Mechanical Properties of Poly(vinyl alcohol) Hydrogels
}

\author{
Koichi YaO, Jun-ichi HorinaKA, and Toshikazu TAKIGAWA ${ }^{\dagger}$ \\ Department of Material Chemistry, Kyoto University, Nishikyo-ku, Kyoto 615-8510, Japan \\ (Received : September 5, 2018)
}

\begin{abstract}
Poly(vinyl alcohol) (PVA) hydrogels were prepared by the repeated freezing and thawing cycles, and creep behavior of the gels was examined. In the gel preparation, the salt $\mathrm{K}_{2} \mathrm{CO}_{3}$ was introduced to obtain the clear gel structure. By the creep we also prepared the condensed PVA gels with unidirectional structure. The mechanical anisotropy was observed for the unidirectional gel.
\end{abstract}

Key Words: PVA gel / Freezing and thawing cycles / Salting-out / Unidirectional gel / Creep

\section{INTRODUCTION}

Polymer gels are composed of polymer networks and solvent molecules, and thus deformation of the gels is affected by the movement of solvent. Nakamura et $a l^{1)}$ have reported interesting deformation behavior for gellan hydrogels under uniaxial compression: Axial compression of cylindrical specimen at a very low deformation rate gives a thin gel disk but the diameter remains unchanged before and after compression. If we introduce the Poisson ratio $(\mu)$, this uniaxial compression corresponds to the special case at $\mu=0$. Here, we have employed the extended definition of $\mu \mathrm{as}^{2)}$

$$
\mu=-\frac{\ln \lambda_{\perp}}{\ln \lambda_{/ /}}
$$

where $\lambda_{/ /}$is the stretch ratio parallel to stretching and $\lambda_{\perp}$ is the stretch ratio perpendicular to stretching. For the uniaxial compression at $\mu=0$, only a very low deformation rate is indispensable because expelling solvent from the gel must require rather long time, suggesting that this compression is attained for any polymer gels. However, to our knowledge, this type of compression appears not to be realized for other gels than gellan hydrogels: An appropriate network structure is also required to achieve the above compression by using a conventional tensile tester. If this type of compression is applied to various polymer gels, we will also have new soft materials with mechanical anisotropy.

Poly(vinyl alcohol) (PVA) gives tough hydrogels: Until now, several methods ${ }^{3-6)}$ have been developed to obtain the

\footnotetext{
$\dagger$ Corresponding author

E-mail : takigawa.toshikazu.7u@kyoto-u.ac.jp

Tel: +81-75-383-2453; Fax: +81-75-383-2458
}

tough hydrogels. Following Nambu's method, ${ }^{3)}$ Watase $e t a l^{4)}$ developed a gelling method composed of freezing and thawing cycles for PVA aqueous solutions. This preparation method is very simple but gives strong hydrogels. The aim of this study is to find out the experimental conditions to realize the above-mentioned compression for PVA hydrogels. The preparation method by Watase et $a l^{4)}$ is modified by cooperating with the salting-out effect ${ }^{7}$ at low temperatures for the smooth compression. Mechanical properties of the compressed hydrogels, which are structurally anisotropic, are also investigated.

\section{EXPERIMENTAL}

Commercial grade of PVA (VM-17, Japan Vam \& Poval Co.) was used in this study. Distilled water was used as a solvent. According to the supplier's data sheet, the average degree of polymerization was 1700 and the degree of saponification ranged from $95 \mathrm{~mol} \%$ to $97 \mathrm{~mol} \%$. PVA hydrogels were prepared by the method of repeated freezing and thawing cycles. PVA aqueous solutions were initially prepared at $95^{\circ} \mathrm{C}$. In most cases, the salt $\left(\mathrm{K}_{2} \mathrm{CO}_{3}\right.$; Wako) solutions prepared beforehand was added to the PVA solutions. The polymer concentration (c) was fixed to be $10 \mathrm{wt} \%$ but the concentration of the salt $\left(c_{\mathrm{s}}\right)$ changed from 0 to $4 \mathrm{wt} \%$. The solutions were then cast into the molds. Two types of molds were used to prepare the gel specimens: One was used to prepare the cylindrical specimens (both diameter and height were $10 \mathrm{~mm}$ ) for the creep tests, and the other to prepare the sheet-shaped ( $5 \mathrm{~mm}$ thick) or cubic specimen (length of one side is $\sim 10 \mathrm{~cm}$ ) for mechanical tests. The mold filled with the PVA solution was kept in the refrigerator (set at $-25^{\circ} \mathrm{C}$ ) for $10 \mathrm{~h}$, and then 
thawed at room temperature $\left(\mathrm{RT} ; \sim 20^{\circ} \mathrm{C}\right.$ ) for $2 \mathrm{~h}$. The above freezing and thawing cycle was repeated three times to obtain the gel specimens.

Firstly, squeezing tests under a constant weight were made at RT to determine a suitable $c_{\mathrm{s}}$, where the compression occurs at $\mu \sim 0$, for the cylindrical PVA gels. The initial height $\left(h_{0}\right)$ and the initial diameter $\left(d_{0}\right)$ of the specimens were commonly $10 \mathrm{~mm}$. The changes in weight and diameter in $6 \mathrm{~h}$ after weight application were examined. The applied weight was $129 \mathrm{~g}$. Then, detailed creep experiments were made at $39 \mathrm{~g}$ for the cylindrical gels at $c_{\mathrm{s}}$ decided $\left(c_{\mathrm{s}}=3 \mathrm{wt} \%\right.$, as will be shown later). The creep process of the samples was recorded by using a video camera and a DVD recorder, and was then analyzed.

To prepare condensed PVA gels $(c \sim 50 \mathrm{wt} \%)$, two condensation methods were used for the PVA gel at $c=10 \mathrm{wt} \%$ and $c_{\mathrm{s}}=3 \mathrm{wt} \%$ : One is simple drying of the sheet-shaped specimen in the air (we call this "iso-gel"; $c=48 \mathrm{wt} \%$ ), and another is uniaxial compression of the cubic specimen under biaxial constrains (this is called "comp-gel"; $c=52 \mathrm{wt} \%$ ). In the latter, $\mu \sim 0$ held and thus obtained gel was anisotropic. The comp-gel was stable (i.e., never going back to the expanded state) enough to apply to the mechanical tests. Mechanical properties of the condensed gels at RT were examined in the tensile mode by using a RTM-500 tensile tester (Orientec) in the air. Rectangular film samples (5 mm width and 1 3 mm thickness) cut from the bulk gel were used for the tensile tests. The initial length between clamps $\left(l_{0}\right)$ was $20 \mathrm{~mm}$ and crosshead speed was $10 \mathrm{~mm} / \mathrm{min}$. Because the comp-gel is anisotropic, the stretching was made in two different directions: parallel and perpendicular to compression. Hereafter, we designate the comp-gel stretched in parallel to compression as "comp-gel (//)" and that in perpendicular to compression as "comp-gel $(\perp)$ ". The stretch ratio $(\lambda)$ was used as a strain measure, and the tensile stress $(\sigma)$ was defined by $\sigma=f / A_{0}$, where $A_{0}$ is the initial force-acting area. Young's modulus $(E)$ was determined from the initial slope of the $\sigma-\lambda$ curve. The tensile tests were also carried out for the isogel and the data were analyzed in the similar way to those of the comp-gel samples.

\section{RESULTS AND DISCUSSION}

As well known, the method of repeated freezing and thawing cycles employed in this study is based on the fact that the formation of ice domains enhances the PVA concentration outside the ice domains at low temperatures and PVA aggregates to form the network in the high $c$ region. ${ }^{3-5)}$ The network structure becomes slightly ambiguous by thawing, but the increased freezing and thawing cycles make the network structure clearer. In this study, $\mathrm{K}_{2} \mathrm{CO}_{3}$ was used as an added salt to shorten the cycle time. The salting-out effect, ${ }^{7)}$ which enhances the aggregation of PVA chains, was not observed even for the highest $c_{\mathrm{s}}(4 \mathrm{wt} \%)$ at RT, but at low temperatures we observed the salting-out behavior. This is because the PVA concentration was enhanced by the ice formation.

Simple squeezing (or creep) tests under a constant weight in the time interval of $2 \mathrm{~h}$ were made for the cylindrical gels to decide the suitable $c_{\mathrm{s}}$. Table I lists the sample code, the change in gel weight $(\Delta w)$ and the change in diameter $(\Delta d)$ of the PVA gel specimens. Here, $\Delta w$ and $\Delta d$ are normalized by the initial weight $\left(w_{0}\right)$ and $d_{0}$, respectively. First two digits in the sample code stands for $c$ in $\mathrm{wt} \%$ and the last one digit does for $c_{\mathrm{s}}$ in $\mathrm{wt} \%$ in the pre-gel solutions. Just after gelation, all gel specimens obtained were opaque, but no syneresis occurred except for the gel at $c_{\mathrm{s}}=4 \mathrm{wt} \%$. By the application of the weight, $\Delta w / w_{0}$ monotonically decreases with increasing $c_{\mathrm{s}}$. Concerning the diameter, $\Delta d / d_{0}>1$ at $c_{\mathrm{s}}$ at $1 \mathrm{wt} \%$ and $2 \mathrm{wt} \%$, but $d / d_{0} \cong 1$ at $c_{\mathrm{s}}=3 \mathrm{wt} \%$. At $c_{\mathrm{s}}=4 \mathrm{wt} \%, d / d_{0}<1$ occurs due to the syneresis. Therefore, we decided the suitable $c_{\mathrm{s}}$ for the compression at $\mu \sim 0$ as $3 \mathrm{wt} \%$.

As stated in the previous section, the PVA gels prepared by the repeated freezing and thawing cycles were opaque. This is because the gels are porous, and the porous structure realizes a relatively fast creep accompanying the volume change. Figure 1 shows the time $(t)$ dependence of $h$ and $d$ of the cylindrical gel at $c=10 \mathrm{wt} \%$ and $c_{\mathrm{s}}=3 \mathrm{wt} \%$ under load. The applied compressional force was $39 \mathrm{~g}$. As can be seen from Panel (a), $h$ monotonically decreases with increasing $t$ and finally reaches to an equilibrium value of $h\left(h_{\infty} ; h_{\infty}=\right.$ $8.6 \mathrm{~mm})$. On the other hand, $d$ remains constant after loading (Panel (b)), suggesting that the creep of the gel occurs at a constant $d$. This means that $\mu=0$ holds for the creep process. The slight increase of $d$ by loading results purely from the elastic deformation of the gel specimen: no squeeze of water occurs just after loading. The trace of this loading effect also

Table I Sample code, the change in gel weight $\Delta w / w_{0}$ and the change in diameter $\Delta d / d_{0}$ for PVA gels. For details see text.

\begin{tabular}{|c|c|c|}
\hline sample code & $\Delta w / w_{0}$ & $\Delta d / d_{0}$ \\
\hline PVA101 & -0.035 & 0.16 \\
\hline PVA102 & -0.045 & 0.11 \\
\hline PVA103 & -0.17 & 0.020 \\
\hline PVA104 & -0.24 & -0.10 \\
\hline
\end{tabular}


emerges on the $h-t$ curve.

In Fig. 2(a), the semi-logarithmic plot of $\left(h-h_{\infty}\right)$ versus $t$ for the PVA gel is shown. Almost all data points fall on a single line. This indicates that $h$ decays as

$$
h(t)=\Delta h \exp \left(-\frac{t}{\tau}\right)+h_{\infty}
$$

and thus $\left(\Delta h+h_{\infty}\right)=h_{0}$. Here, $\Delta h$ is a constant and $\tau$ is the time constant characterizing solvent squeezing. We have $\tau=7.0 \times 10^{3} \mathrm{~s}$ for the PVA gel examined. For the solvent squeeze by the axial compression of the cylindrical gel we have

$$
-\frac{\mathrm{d} V}{\mathrm{~d} t}=S v
$$

where $V$ is the volume of the gel, $S$ is the lateral area of the gel, and $v$ is the solvent velocity at the surface. We assume here that the solvent is squeezed only from the lateral area. If the compression occurs at $\mu=1$, then we have $V=\pi d_{0}{ }^{2} h / 4$
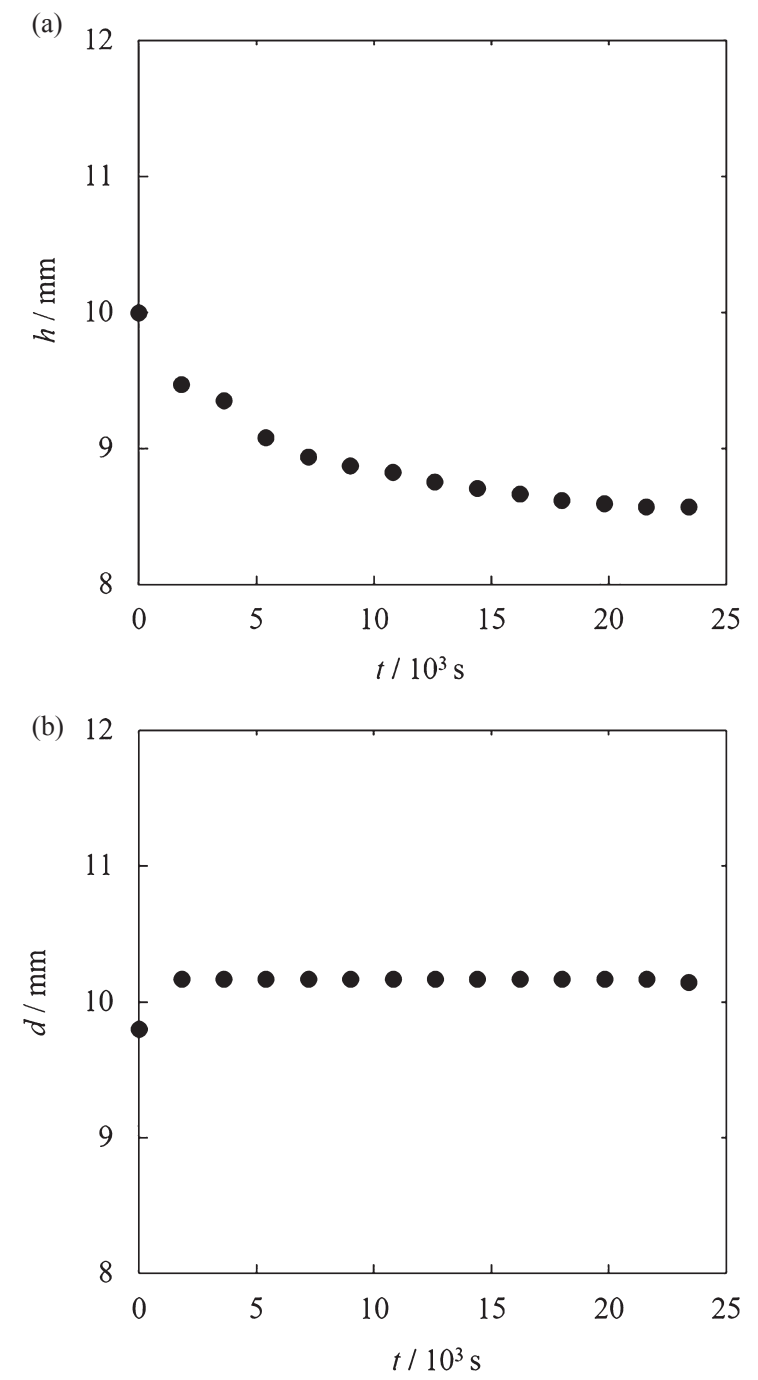

Fig. 1 Time $(t)$ dependence of (a) height $(h)$ and (b) diameter $(d)$ of the PVA gel in the course of creep. and $S=\pi d_{0} h$. Thus, Equation (3a) is re-written by

$$
\frac{\mathrm{d} \ln h}{\mathrm{~d} t}=-\frac{4}{d_{0}} v
$$

Combining Equations (2) and (3b), we know that $\tau$ is related to the initial solvent velocity $v_{0}$ (i.e., $v$ at $t=0$ ) as $\tau=\Delta h / 4 v_{0}$ if $d_{0}=h_{0}$.

The creep compliance $(J)$ is defined by $J=\varepsilon / \sigma_{0}$, where $\varepsilon$ is the strain given by $\varepsilon=\left(h-h_{0}\right) / h_{0}$ and $\sigma_{0}$ is the stress converted from the compressional force (i.e., $39 \mathrm{~g}$ ). It should be noticed that $\sigma_{0}$ should be negative. Figure 2(b) shows the plots of $J$ against $t$ for the PVA gel. The limiting value of $J$ at $t \rightarrow \infty\left(J_{\infty}\right)$ can be estimated as $J_{\infty}=2.8 \times 10^{-4} \mathrm{~Pa}^{-1}$ for the PVA gel. When the compressed gel after measurement was immersed in a distilled water, the gel anisotropically reswelled to some extent ( $h=8.6 \mathrm{~mm}$ to $9.1 \mathrm{~mm}$ ). Namely, $h$ increased in water with keeping $\mu \sim 0$.

Figure 3 shows the stress-strain curves of the condensed PVA gels at $c \sim 50 \mathrm{wt} \%$. The stress level for "iso-gel" is the
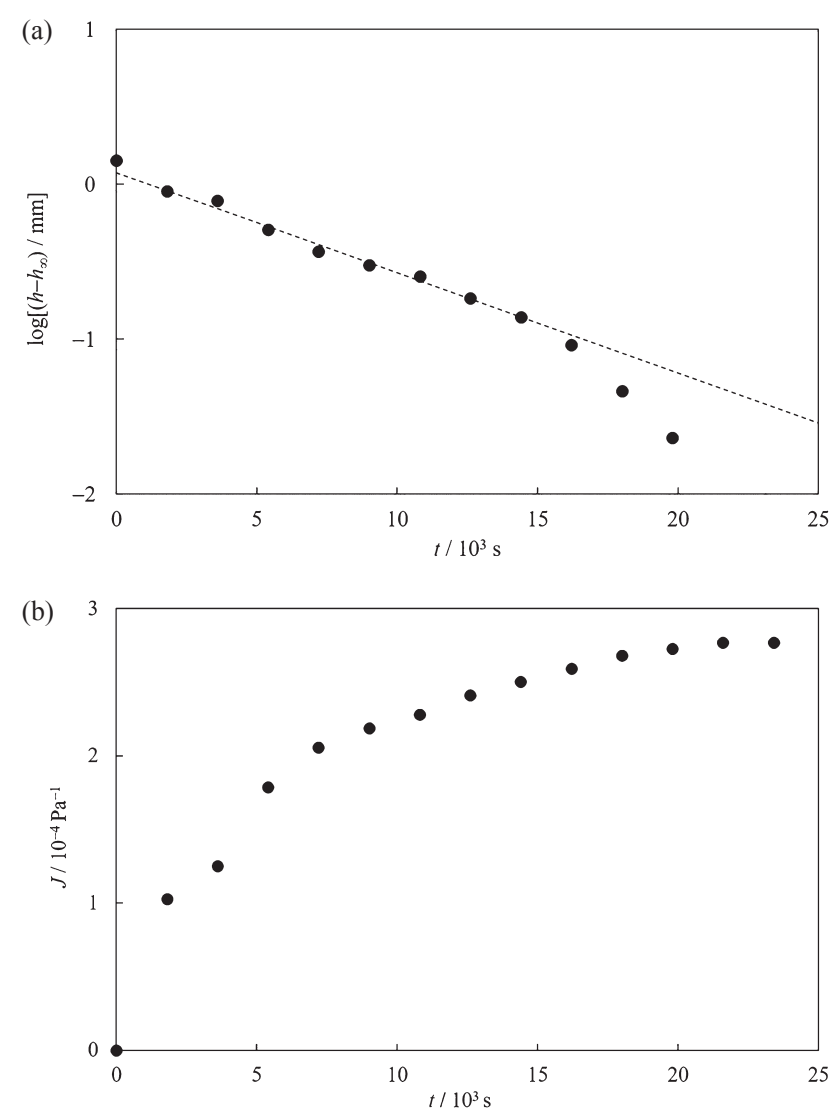

Fig. 2 (a) Semi-logarithmic plots of $\left(h-h_{\infty}\right)$ vs time ( $\left.t\right)$ for the PVA gel: (b) Creep compliance $(J)$ plotted against time $(t)$ for the PVA gel. 
highest over the entire range of $\lambda$, and a clear yielding is also observed for the iso-gel. This yielding must occur in the PVA domains in the gel, as is the case of PVA gels swollen by alcohols or hydrogels including microcrystalline domains. ${ }^{8)}$ For the comp-gel, the $\sigma-\lambda$ curve for the comp-gel $(\perp)$ stays in the higher stress side than that for the comp-gel (//), but yielding becomes ambiguous (or, small) for both specimens. The ambiguous yielding may be because the comp-gel experiences the yielding in the course of compression process. ${ }^{9}$ The iso-gel shows the highest $E\left(=2.1 \times 10^{7} \mathrm{~Pa}\right)$ and the comp-gel $(\perp)$ the lowest $E\left(=6.0 \times 10^{5} \mathrm{~Pa}\right)$, and $E$ of the comp-gel $(/ /)$ is located between $\left(E=1.1 \times 10^{6} \mathrm{~Pa}\right)$. Hereafter, we write $E$ of the comp-gel (//) as $E_{/ /}$and $E$ of the comp-gel $(\perp)$ as $E_{\perp}$. Why the comp-gel shows lower $\sigma$ (not only $\sigma / /$ but also $\sigma_{\perp}$ ) than that of the iso-gel may also originate from the fact that the comp-gel experiences the yielding in the preparation stage. In Fig. 4 , the ratio of $\sigma_{\perp}$ to $\sigma_{/ /}\left(\sigma_{\perp} / \sigma_{/ /}\right)$is plotted against $\lambda$. Here, $\sigma_{\perp}$ is $\sigma$ of the comp-gel $(\perp)$ and $\sigma_{/ /}$is $\sigma$ of the comp-gel $(/ /)$. At small $\lambda,\left(\sigma_{\perp} / \sigma_{/ /}\right)$stays around 1.8, which means that $E_{\perp} / E_{/ /} \sim 1.8$, and then increases with increasing $\lambda$. The absolute values of $E_{/ /}$and $E_{\perp}$ are high enough compared with $J_{\infty}^{-1}$. This may be because the $c$ region of the gel for the creep measurement is low and $J_{\infty}^{-1}$ also reflects the compression process at long times giving $\mu \sim 0$.

Figure 5 schematically illustrates the structures of the PVA gels examined in this study, where the gels are treated as the porous materials. It is noticed that the pores in the gels are actually interconnected. The as-prepared gel corresponds to the gel just after the freezing and thawing cycles are finished. In the comp-gel, the pores are deformed to be ellipsoidal: The ellipsoids are made of the spheres in the as-prepared state purely the vertical compression in the preparation stage because $\mu \sim 0$ in the preparation stage. This suggests that the comp-gel is unidirectional and already undergoes yielding in the compression process. On the other hand, the iso-gel is made of the as-prepared gel by the isotropic shrinkage, so that the iso-gel remains isotropic and does not experienced yielding. The comp-gel $(/ /)$ and the comp-gel $(\perp)$ correspond to the stretched states of the comp-gel. As stated previously, the $\sigma-\lambda$ curves of these gels showed ambiguous yielding. This may be because the comp-gel already experienced yielding in compression, as stated previously. Concerning the modulus of the comp-gel (//) and the comp-gel $(\perp), E_{/ /}$was smaller than $E_{\perp}$. This originates from the lower value of the Poisson ratio for the comp-gel (//), as below. Let $\mu_{\perp}$ be $\mu$ obtained in the elongation for the comp-gel $(\perp)$ and $\mu_{/ /}$be that for the comp-gel $(/ /)$, and the Maxwell-Betti reciprocal theorem gives ${ }^{10)}$

$$
\frac{\mu_{\perp}}{E_{\perp}}=\frac{\mu_{/ /}}{E_{/ /}}
$$

This equation says that the lower modulus comes from the lower Poisson ratio. For the comp-gel (//) showing lower $E_{/ /}$, the horizontally oriented pores must change to the vertically oriented ones, which may lead to the insufficient contraction giving lower $\mu$. On the other hand, no change in shape is not needed for the comp-gel $(\perp)$; vertically oriented to vertically oriented. This change must be easier and thus gives higher value of $\mu$ rather close to $1 / 2$.

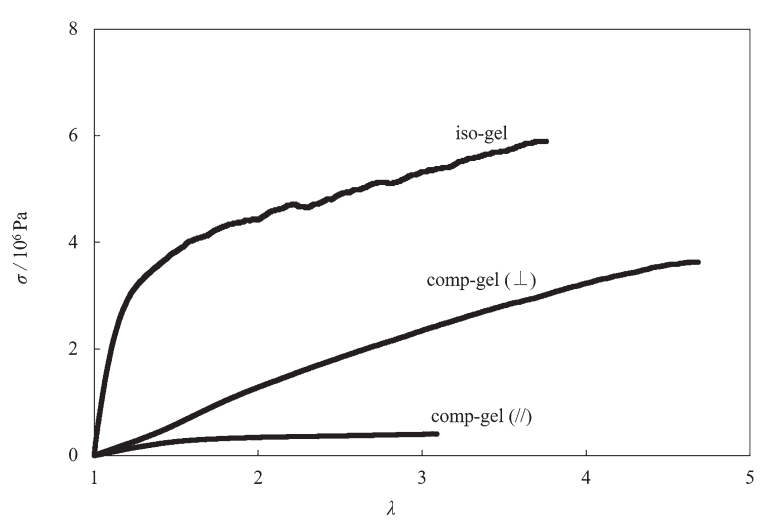

Fig. 3 Stress $(\sigma)$ - stretch ratio $(\lambda)$ curves for the condensed PVA gels.

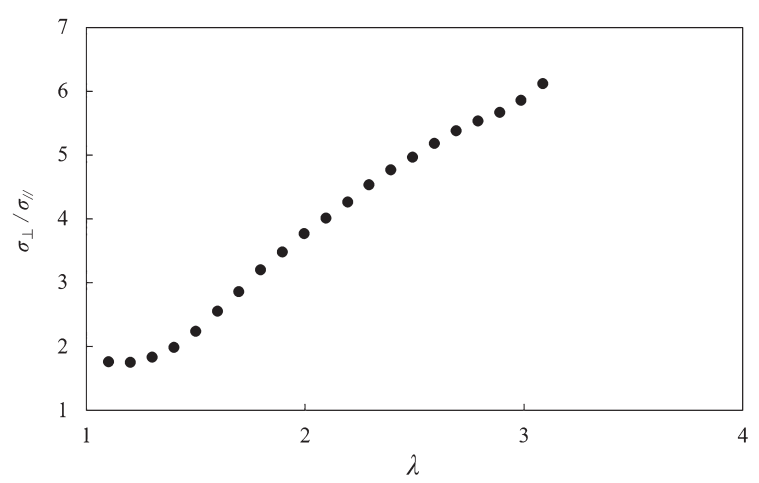

Fig. 4 Stress ratio $\left(\sigma \perp / \sigma_{/ /}\right)$plotted against stretch ratio $(\lambda)$ for the comp-gel.

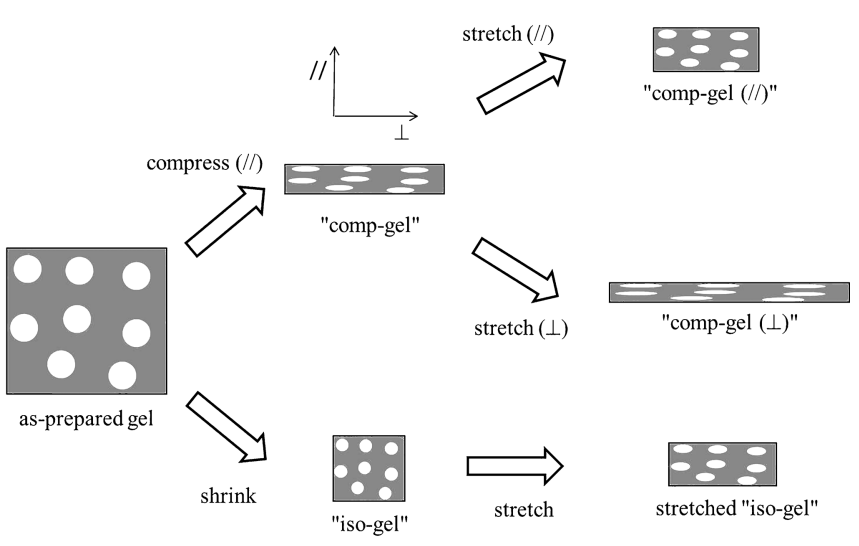

Fig. 5 Schematic representation of the PVA gels examined in this study. 


\section{REFERENCES}

1) Nakamura K, Shinoda E, Tokita M, Food Hydrocolloids, 15, 247 (2001)

2) Yamamoto M, Buttai no Henkeigaku, Seibundoshinkosha, Tokyo (1972).

3) Nambu M, Japanese Patent, 82-130543 (1982).

4) Watase M, Nishinari K, Nambu M, Cryo Lett, 4, 197 (1983).

5) Nagura M, Hamano T, Ishikawa H, Polymer, 30, 762 (1989).

6) Hyon S-H, Cha W-I, Ikada Y, Polym Bull, 22, 119 (1989).
7) Dai L, Ukai K, Shaheen SM, Yamaura K, Polym Int, 51, 715 (2002).

8) Takigawa T, Kashihara H, Urayama K, Masuda T, Polymer, 33, 2334 (1992).

9) Takigawa T, Kashihara H, Masuda T, Polym Bull, 24, 613 (1990).

10) Ehrenstein GW, Faserverbundkunststoffe: WerkstoffeVerarbeitung-Eigenschaften, Carl Hanser, München (2006). 